\title{
Designing a Sensor Glove Using Deep Learning
}

\author{
Jeremy Chan \\ Graz University of Technology \\ jchan@know-center.at
}

\author{
Eduardo Veas \\ Graz University of Technology \\ eveas@tugraz.at
}

\author{
Jörg Simon \\ Know Center $\mathrm{GmbH}$ \\ jsimon@know-center.at
}

\begin{abstract}
When designing a smart glove for gesture recognition, the set of sensors available and their layout on the glove are crucial. However, once a computational model reaches acceptable recognition accuracy, it is often not clear which sensors are more important for the task. Nor whether some sensors can be strategically removed while retaining similar performance in order to save cost. Furthermore, when aiming for a personalized setup, there can be minor deviation in how gestures are performed by each participant, and so the importance of a sensor may vary between participants. In this paper, we use feature selection to explore whether a personalised glove can be produced, and whether the set of significant sensors persist between users. We present a deep learning algorithm which utilises a layer of weights to estimate the importance of each sensor in relation to each other. Besides estimating importance in relation to recognition accuracy, it is demonstrated how the importance estimates can be extended to take into account factors external to the computational model, such as costs. This allows for a cost effective elimination of sensors to reduce hardware redundancy whilst having a controlled impact on performance. We provide 2 methods: generic or specific. The generic method exploits the importance estimate from all participants to select a set of sensors for removal. Whereas the specific method estimates importance, and removes sensors based on individuals to provide a personalised setup.
\end{abstract}

\section{CCS CONCEPTS}

- Human-centered computing $\rightarrow$ Gestural input; • Computing methodologies $\rightarrow$ Neural networks; Feature selection; • Hardware $\rightarrow$ Sensor devices and platforms.

\section{KEYWORDS}

Sensor selection;Wearable design;Computational design;Gesture detection, Deep Feature Selection, Deep Learning

\section{ACM Reference Format:}

Jeremy Chan, Eduardo Veas, and Jörg Simon. 2021. Designing a Sensor Glove Using Deep Learning. In 26th International Conference on Intelligent User Interfaces (IUI '21), April 14-17, 2021, College Station, TX, USA. ACM, New York, NY, USA, 11 pages. https://doi.org/10.1145/3397481.3450665

\section{INTRODUCTION}

Humans use hands as the main means of interaction with the surrounding environment and the entities therein. Hands are therefore

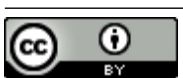

This work is licensed under a Creative Commons Attribution International 4.0 License

IUI '21, April 14-17, 2021, College Station, TX, USA

(c) 2021 Copyright held by the owner/author(s).

ACM ISBN 978-1-4503-8017-1/21/04.

https://doi.org/10.1145/3397481.3450665 a target for computer interfaces [5]. Beyond conventional hand operated devices like the mouse and keyboard, wearable computing has witnessed the development of numerous glove-based systems. For instance, motion capture for graphics animation [27], assessing hand function in rehabilitation [36], or serve as a general purpose input devices [15, 37]. The original aim of gesture recognition was to build computational systems that could detect and translate sign language $[11,23,28]$. But recently, it became the basis for gesture based interaction, for example, using a vocabulary of gestures to interact with a computer $[12,14]$, to control a TV [24], to serve as input for gaming $[13,19,34]$, or to act as a controller in virtual and augmented reality $[17,29,32]$. There are glove-based systems that achieve decent accuracy for vocabularies of up to 30 gestures, either using commercial [21], or custom built gloves [18]. But it is difficult, if not impossible, to trace which sensors are important for the algorithm and which can be eliminated to reduce complexity and costs. Our goals are to reduce the complexity of hardware whilst retaining accuracy, and to find personalised design layout for each user. We also aim to support the designer in realising the range of gestures that can be supported, the accuracy that can be expected and the effectiveness of the hardware from the glove design. We propose a data-driven approach to answer:

(RQ1) Is it possible to remove sensors based on prediction accuracy, to create a minimal design? Is it possible to remove sensors based on reducing cost to create a cost effective design?

(RQ2) Is it possible to personalise the gesture glove to an individual? How does it differ from the generic solution?

(RQ3) Does the set of sensors retained differ when optimising for prediction accuracy-and-costs compared to prediction accuracy only?

A glove-based system or smart glove is a complex, self sustained system, composed of a collection of sensors, electronics and power supply. They are attached to a support worn on the user's hand with embedded data acquisition and processing capabilities [5]. The design of a smart glove requires careful consideration of the hand physiology, the placement of sensors, and the selection of appropriate algorithms to detect gestures. The human hand contains actuators that account for 25 degrees of freedom (DoF), as well as sensors such as thermoreceptors, mechanoreceptors, pain and kinesthetic sensors [30]. A straightforward design would assign one sensor per DoF. But designs have been proposed with varying sensor technology and range of information. However not all of the information offered are significant, and it is difficult to estimate the importance of each sensor for the algorithm at design time. We propose feature selection to estimate the importance of each sensor, allowing for relative comparison and evaluation. This can provide informative insight towards the design of the glove in order for it to be effective as intended.

The model for gesture recognition can be realised with a traditional machine learning classifier, following the extraction of 
hand-crafted spatial and temporal features [18]. But they can also be attained with deep learning classification algorithms. Deep learning algorithms use a cascade of non-linear computational units that learn of hierarchical representations within the data. This allows deep learning classification models to learn the features and their association with the output during training [4]. One disadvantage of deep learning is that the models cannot be inspected easily. With current existing methods, it is difficult to explain the relationship between input sensors and output. It is impossible to explain which part of the input is meaningful towards the output.

The main contribution of this work is an approach using feature importance estimates as a basis to analyse the design of the wearable in relation to gesture recognition. We demonstrate a method to extend importance estimates to external factors that can be represented as a cost, thereby reformulating the task as a utility problem based on accuracy as well as other factors (e.g. economical costs, energy). This method supports the wearable computing designer in either creating a general set up for the majority with gestures of expected accuracy and available budget, or creating a personalised set up for a specific individual. Furthermore, our approach presents a contribution towards inspecting and explaining deep learning model for the decision making of how significant a sensor is towards each setup.

\section{RELATED WORK}

The design of a smart glove for gesture recognition involves hardware design and classification methods. Hardware design is based on knowledge of anatomy and physiology of the hand. In this regard, Cobos had developed a mathematical model to capture the kinematics of the hand[3]. The full model has $24 D O F$, but it also shows that a model with only $9 D O F$ can simulate a correct hand movement with an error of 5\%. Therefore it is necessary to capture the minimum amount of DOF, in order to fully capture the physical movement of the hand [5].

The Sayre glove, developed by de Fanti and Sandin, tracked each finger using a flexible tube with a light source and a photocell [27]. Alternatively, Zimmerman developed an optical flex sensor using optical fibers and deployed it with a data glove [37], which was commercialised as VPLDataGlove later. Currently, various commercial data gloves favor flexion sensors to capture finger joint motion. The CyberGlove II from CyberGlove Systems ${ }^{1}$ employs: two bend sensors per finger, four abduction sensors, sensors measuring thumb crossover, palm arch, wrist flexion and wrist abduction (22DOF). Virtual Labs ${ }^{2}$ offers a range of data gloves equipped with bend sensors on the fingers, 9 DOF orientation sensors for hand and wrist (up to $32 D O F$ ). In contrast, our initial design includes both flex and motion sensors (gyroscope + accelerometer) per finger to capture gestures.

Our goal is to use numerical methods to optimise the design and models of the smart wearable system, thus we concentrate on glove based systems for gesture recognition. Using the VPLDataGlove, Murakami and Taguchi describe a recurrent neural network (RNN) approach to detect postures corresponding to 42 alphabet letters

\footnotetext{
${ }^{1}$ http://www.cyberglovesystems.com

${ }^{2} \mathrm{http}: / /$ www.virtualmotionlabs.com/
}

with accuracy ranging from $92.9 \%$ to $94.3 \%$ [20]. However, attempting to predict Japanese sign language gestures composed of those letters required pre-wiring the hand position in the network to deal with the time series. With this approach they reported $96 \%$ accuracy on 10 words [20]. Xu et al. use a Cyberglove (18 DOF) and a feedforward neural network (FFNN) to distinguish 15 gestures with an accuracy of $98 \%$ [33]. Neto et al. also used a FFNN, but a CyberGlove II instead (22 DOF) to construct a hand gesture recognition system for interacting with robots, achieving an accuracy of $99.8 \%$ on 10 gestures and 96.3 on 30 [21]. Romaszewski et al. used a list of 22 natural hand gesture. They first re-sampled and interpolated data, then applied LDA to discriminate the re-sampled segments with an accuracy of $92.8 \%$ [25]. However, in a live recognition system, this re-sampling and interpolation is not possible.

Luzhnica et al. followed a human activity recognition (HAR) approach, and used data from a custom built sensor glove to classify 31 gestures [18]. They used statistical and spectral (FFT) features obtaining $98.5 \%$ accuracy. This paper uses the same data set to personalize the 52 DoF data glove. The focus of this paper is the use of feature selection to determine i) the essential sensors for the overall group, or ii) a single individual, iii) in a cost-effective manner.

A major challenge in modelling is knowing what features are important and which are redundant. Because this information is usually not known a priori, we tend to use all features despite the increased imposed complexity [26]. To reduce this issue, feature selection is often used. Feature Selection is the process of selecting and removing pieces of information, features, from all available information, the search space [8]. A common approach is the use of regularisation, with the $\ell_{1}$ norm (LASSO), $\ell_{2}$ norm (Ridge), or both $\ell_{1}$ and $\ell_{2}$ (Elastic Net) [1]. Neural networks (NN) process features with their hidden layers, so the selection happens internally. But this limits the comprehension of each feature's relevance with respect to the output of NN [35].

There exists approaches based on network pruning to determine relevance by setting the weights of layers to zero whilst minimising changes in the error rate of the $\mathrm{NN}[31,38]$, this would however, require an extensive analysis of hidden layers [8]. To understand the selection process in $\mathrm{NN}$, interpretability methods have been developed in an attempt to estimate the influence of a given input feature to the prediction made by a model [10]. Roy et al. [26] analysed the activation potential of the first layer connected to the input, to select important features.

Alternatively, Yifeng et al. [16] suggested a method to select important features at the input level from NNs. Their data consisted of a lymphoblastoid cell line sampled into 93 features, where they classified 3 classes: active promoter, active enhancer, and background. Their method works by providing a sparse one-to-one layer between input and the first hidden layer of a NN. Where this layer would automatically selects a subset of the input features during training. Afterwards, they analysed the selected features for any relation, however this required prior knowledge on the subject, which is not always readily available. A variation of this concept by Ning Gui et al.[7], employs a detachable NN between the input and the model, where they attached an attention network to a trained NN. The detachable NN, the attention network, selects features 
from the input using the correlation within to generate probability for each feature.

Our proposed approach also employs a detachable mechanism, but instead of using a NN, a fully connected layer of hidden units is used for analysis. The layer is capable of identifying any relation within the input without any prior knowledge of the data, thus avoiding the need to consult experts. It is also capable of ranking features in terms of significance through using the weights of the layer as an estimate of importance. Furthermore, existing methods can only feature select based on the objective function of the $\mathrm{NN}$, which is the maximisation of accuracy for regression or classification. The proposed method allows including other constraints in the feature selection process, specifically we introduce an additional cost-function for the hardware used. We offer thus, a numerical approach of using deep learning to optimise the hardware of a gesture glove and the recognition of the gesture classes. This allows for the feature selection to be based on cost-effectiveness rather than just the maximisation of prediction accuracy.

\section{PRELIMINARIES}

For the work conducted in this paper, we used the dataset obtained from Luzhnica et al. [18] and therefore their experimental glove design. However instead of using traditional machine learning algorithms, we deployed the use of a deep learning algorithm as basis for our explainable algorithm. This section aims to provide the necessary details of their glove design and their data to interpret the results of sensor selection obtained with our algorithm.

\subsection{Sensor Glove Hardware}

The glove was designed to fit as many sensors as possible for data studies, even if it meant delivering redundant information. Figure 1 shows the schematics, as well as the finished glove prototype. It includes a total of 28 sensors, counting 63 data channels on the hand, including: a) five fingertip pressure sensors, b) thirteen (13) flex sensors, c) seven (7) six DoF (6DoF, accelerometer and gyro, both with $\mathrm{X}, \mathrm{Y}, \mathrm{Z}$ axes) IMUs, and d) one magnetometer. The IMUs operate at 2.3 to $3.4 \mathrm{~V}$ and send data over $I^{2} \mathrm{C}$. The chip sensitivity was set to the lowest setting for of each accelerometer $(+/-2 g)$ and gyro $(+/-250 \circ / s)$. The sensors and data channels mapping is shown in Table 1.

The sensor glove captures movement in $52 \mathrm{DoF}$. Two flex sensors capture wrist $u p$ and down movements $(2-D o F)$. One 6 DoF IMU is placed on the palm, and a magnetometer $3-D o F$ on the back of the hand. Two flex sensors $2 \mathrm{DoF}$ and one IMU $6 \mathrm{DoF}$ on each fingertip capture fingers. The thumb has three flex sensors (3 DoF) and a 6 DoF IMU.

An Arduino Due board controls the data acquisition. The IMUs and magnetometer are directly connected to $I^{2} C$ bus, as well as a multiplexer, which cycles over the analogue sensors (flex and pressure) every $12 \mathrm{~ms}$. A C program on the Arduino initialises the sensors on startup and transmits the data every 12 milliseconds $(83.3 \mathrm{~Hz})$ over USB serial connection to a PC. The sampling frequency is well above the frequency of human movements, < $20 \mathrm{~Hz}$ [2].
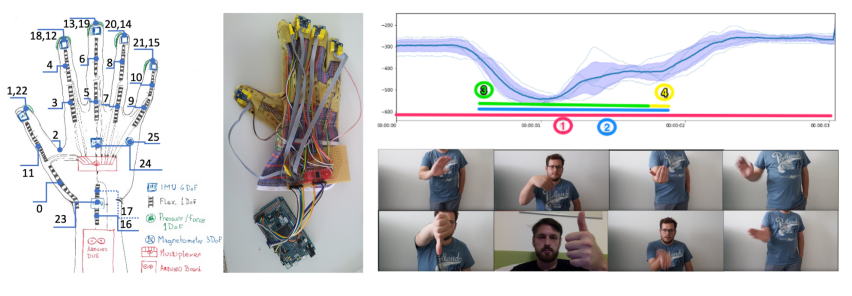

Figure 1: Left. Sensor Glove Hardware: A construction glove with 28 sensors, which has 63 channels of data. The labels are the sensors id. Right Top. Gesture Window Labeling. (1) Automatic, (2) Manual, (3) Dynamic, (4) Static. Right Bottom. Eight example gestures.

\subsection{Gestures and Data}

The dataset consists in a compendium of 31 natural appearing gestures obtained from 22 participants (12 male and 8 female, aged $24-40$ years). They are acquired with the sensors of the glove.

A script was used to control the collection of data from all channels of the glove at a fixed frequency. The process for collection of a single gesture consisted of: i) watching a video of the gesture performed 3 times. ii) a single trial of the gesture. iii) a countdown shown on the screen iv) a window of 3 seconds, for the participant to perform the gesture (trial) v) repeating steps (iii) and (iv) a number of times ( 5 or 10, agreed at the beginning). Each $3 \mathrm{~s}$ window was automatically annotated by the data collection script. Furthermore, the experimenter annotated the dynamic part of the gesture (where the arm is moving) and the static part (where the gesture is fixed), during gesture performance.

Fig 1 illustrates the hierarchy of labels for a $3 s$ trial. Each trial has one automatic label, one manual label, one dynamic, and one static label. Automatic consists of the annotated start and end time defined by the script. Manual consists of the start and end time determined by the observer. Static represents the start, when the hand is still, and when a gesture ends, when the hand rests again. And dynamic represents the start and end time of the motion performed.

\subsection{Modelling: Deep Learning Gesture Recognition}

Aiming at reducing reliance on pre or post processing of features, we use deep learning to address the gesture recognition problem. With classification models, they can innately learn the features and their association with the output through the hidden units within the layers of the model.

Data stream processing: The objective is to detect patterns within the data stream of the sensors that correspond to gestures [22], where the data stream is a multivariate time series. The manual labels were found more suitable for the classification task. Furthermore, the data is serialised into windows through a sliding window approach[6]. Concretely, a window size of 64 was used as this is found to be better in capturing the patterns of the gestures.

Training and validation datasets: $K$-fold cross validation, where $K$ is the number of participants, was used for the training and testing of the classification models. This is a leave-1-out approach that creates the training data through the gesture data of all but one participants, and the gesture data of the left-out participant is used 


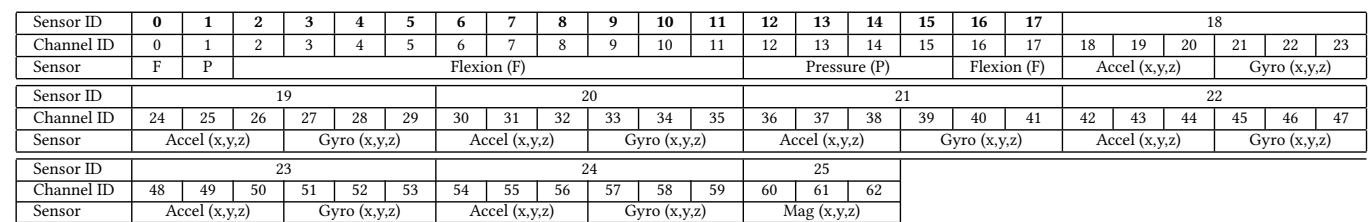

Table 1: Sensors and Data Channels Mapping. Accel stands for the Accelerometer and Gyro for the Gyroscope part of an IMU. Mag for a Magenetometer. The positions of sensors are shown Table 1: Sens 1

for validation. This is to maximise the model's ability to generalise and avoid overfitting. The 22 -fold cross validation was repeated 3 times for each participants,as demonstrated in 2 . The focus of the evaluation has been placed upon the true positive rate (TPR) for each gesture, as they are more informative in comparison to measures of accuracy.

Architecture: A recurrent neural network is used because it has the capability to make use of temporal characteristic from the full sequence of data, which is crucial in distinguishing between gestures. Concretely, Long-short-term memory recurrent neural networks (LSTMs) are recurrent networks that include a memory to model temporal dependencies in time series problems. They are able to remember information for an arbitrary number of time steps [9]. The model consists of 2 layers. A fully connected layer with 256 LSTM cells and 128 hidden units along with a tanh activation. And a fully connected softmax output layer to perform classification. The model uses the Adam optimizer with a learning rate of 0.0001 . These parameters and the pre-processing (window size) were fine-tuned over numerous iterations.

Results: On average, 10 epochs of training are required for the model to reach $90 \%$ in accuracy, precision, recall and F-measure. However, Figure 2 shows that despite the high TPR for the majority of the gestures and participants, there still exists some gestures for some participants that are sub par in TPR. For example gesture 21 of participant AW18. There are also a small number of participants who generally have a lower TPR than most across the runs, i.e PC29.

\section{DEEP FEATURE AND SENSOR SELECTION}

The basis of the method is from Yifeng et al.[16] and Ning Gui, et al.[7], introducing a sparse layer between the input and the first hidden layer of a neural network, to then perform additional processing for the model to select a subset of features during training. Our approach expands upon such in two areas: feature analysis and feature selection. It is further extended to take into account factors that are external to the data, in this case the cost of hardware.

\subsection{Methodology}

A prerequisite of the method we employ is an existing neural network model that could accurately and reliably predict using all the data channels provided from the sensors. This existing model will serve as the baseline measure. Its architecture is described in Section 3.3. A prerequisite is that any models using the introduced method of deep feature selection should not be performing worse than the baseline measure. To make sure of this, we mainly use TPR from the gesture predictions, as well as the metric scores to evaluate the models.
4.1.1 Training. This method introduces a layer of weights between the input and the first hidden layer of the model. This is a sparse layer of $1 \mathrm{~s}$ with a bijective relation to each data channel of the sensors, it will be referred to as the importance estimate layer, $\boldsymbol{I E}$ layer, see Left of Figure 3.

With this layer, we propose a method of training that requires two phases. This is demonstrated in the Right of Figure 3. In the initial phase, the IE layer is set to immutable which means the weights of the layer will not undergo any changes. This allows for the model to train with all data channels and their original value, to create a baseline for comparison and for the subsequent phase.

For the 2nd phase, the baseline from the initial phase is used. The $I E$ layer is set to trainable and everything else from the model is set to immutable. With this, the training continues. This allows the weights of the $I E$ layer to adjust accordingly toward the objective function of the model, the minimisation of the loss function. As the 2nd phase is completed, there will be some discrepancy between the weights in the sparse $I E$ layer. This discrepancy provides insight into the significance of each data channel in relation to the loss function, which in this case, is the maximisation of prediction accuracy for the classification of gestures.

4.1.2 Feature Analysis and Selection. For feature analysis, our method analyses the weights of the $I E$ layer. We have extended the existing analysis with a way to rank the relative significance of each feature. And we provide a way to verify by training new models using only the selected features.

Because the weights from the $I E$ layer has a 1-to-1 relation to the data channels of the sensors, and the weights were initialised to 1 in the beginning. The difference in the weights after training can be interpreted as a difference in importance in relation to the loss of the model, which can be used to establish a ranking between the weights. It is important to note that some sensors provide multiple data channels, and in order to conform the estimates, any sensors that provide multiple data channels share the same weight across the multiple data channels throughout the method, an illustration is included in Figure 3 to demonstrate this. Doing this trivialise the importance of each individual data channel of the sensor, however it is necessary in order to rank each sensor in relation to each other. The weights are ranked based on their value, where a higher value would equate to higher importance, and vice versa. Although trivial in nature, our studies found this approach to be effective in selecting data channels for removal. If the aim was to remove $X \%$ of data channels, this can equate to removing $X \%$ of the lowest weights in the $I E$ layer.

After a selection is made based on the assumption for the value of the weights. The next step is to verify the robustness and soundness of the method. To do so, new, reduced models were trained solely 

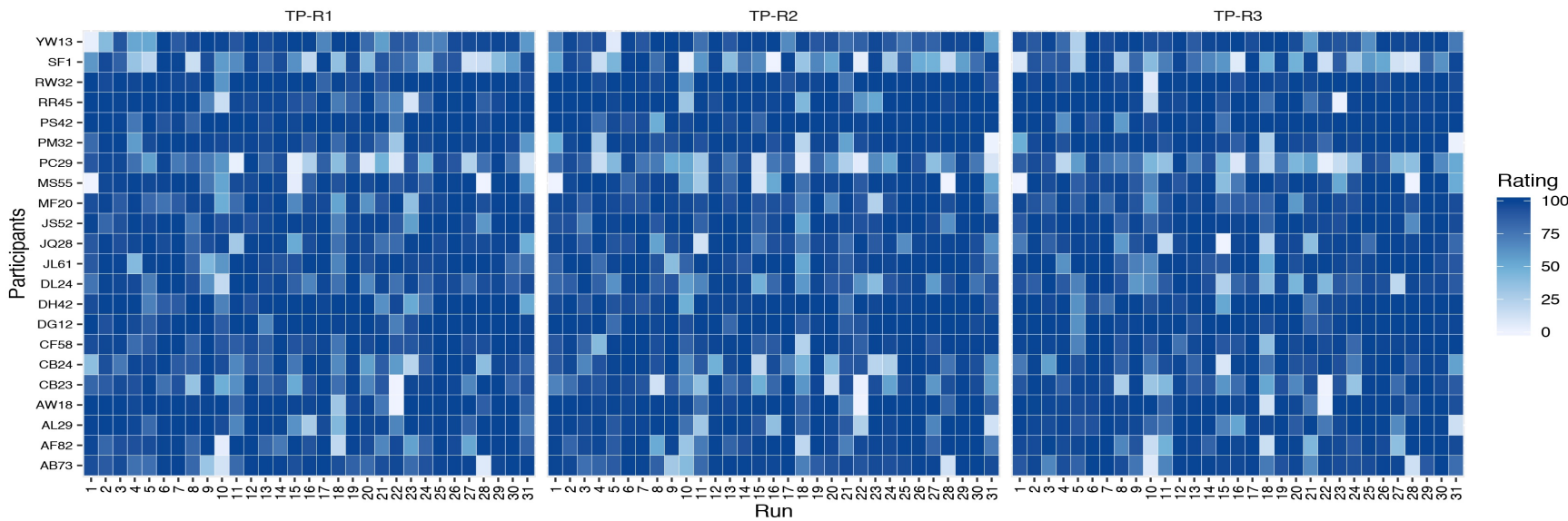

Figure 2: Illustration of the True Positive Rate for each gesture across 3 runs.
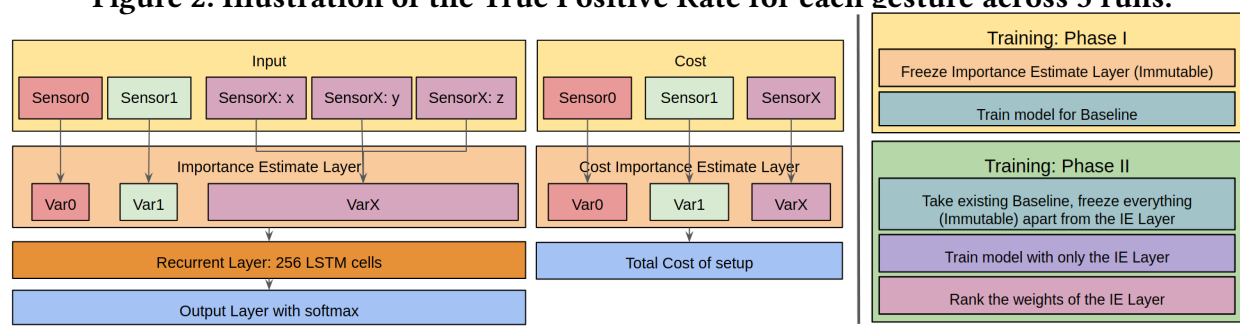

Figure 3: Left shows the set up of the model architecture, illustrating how the weights of the importance estimate layer, $I E$ layer, correspond to each data channel of sensors, variable sharing with the cost importance estimate layer, CIE layer. Right shows the two training phases of the proposed method.

with the selected data channels. This is done by adjusting the value of the supposedly significant channels to 1 in the $I E$ layer weights, and the discarded channels to 0 . After the necessary adjustment, the $I E$ layer is set to immutable for training. Afterwards, the TPR of the gestures can be compared to the existing baseline, where one can conclude if the assumption holds or not.

With the basic of the method outlined, it can be developed even further to either provide a generic design for all participants or a personalised designs of the gesture glove. In order to do so, it is necessary to consider what sensors are important for a single participant. To do so, sensor selection is performed based on the weights of the $I E$ from the results of that participant at face value. An example is demonstrated in Figure 4.

The generic case needs to consider the results from a collection, i.e, from all participants. To do so, all the weights of the $I E$ from each participant have to be taken into account to perform sensor selection, illustrated in Figure 4, where all the weights from each participant were averaged.

4.1.3 Training with relation to the cost of the sensors. The current ranking method is sufficient to reduce the number of channels, but it does not account for the costs of sensors. The method can be extended to consider each individual sensor cost in relation to the data channels they provide with minimum changes to the current framework. In order to incorporate sensor cost, the cost of each sensor needs to be defined. This should reflect upon the monetary cost, as well as the usefulness of the information it is capable of providing, in order to serve as the cost function.
With the cost function established, another sparse layer of weights, initialised to $1 \mathrm{~s}$, can be added to the model. In this case, the weights will have a bijective relation with the cost of each sensor. This layer will also be referred to as cost importance estimate layer, CIE layer, see Figure 3. It has the same principle as the $I E$ layer, where it is concerned with an objective function, in this case, the minimisation of cost for the sensors employed. The layer has the additional purpose of connecting the cost of each sensor and the significance of each sensor's respective data channel(s) together.

The reason another sparse layer is required is because some sensors have multiple data channels, and a single $I E$ cannot take the relation between the data channels and their respective sensor into account. The additional IE, the CIE layer, achieves this by sharing variables between data channels of the same sensor, thus also sharing the same variables with the $I E$ layer, see Figure 3 . With the $I E$ layer maximising classification prediction for gesture, and the CIE minimising cost. This allows for the weights that correspond to the sensors to represent their cost effectiveness.

The phases of training remain the same. In the initial phase, the $I E$ layer is still immutable, but because the variables are shared with the CIE layer, the CIE layer is also effectively immutable. In the 2nd phase of training, the variables of the $I E$ layer and $C I E$ layer are set to trainable where they can adjust accordingly to the objective of the model. Which is now the maximisation of prediction accuracy for the classification of gestures whilst also minimising the cost of sensors used. This is made possible by combining the loss from cross entropy of the predictions with the average cost of the sensors. After training, the sensor selection analysis can be carried out as before. 


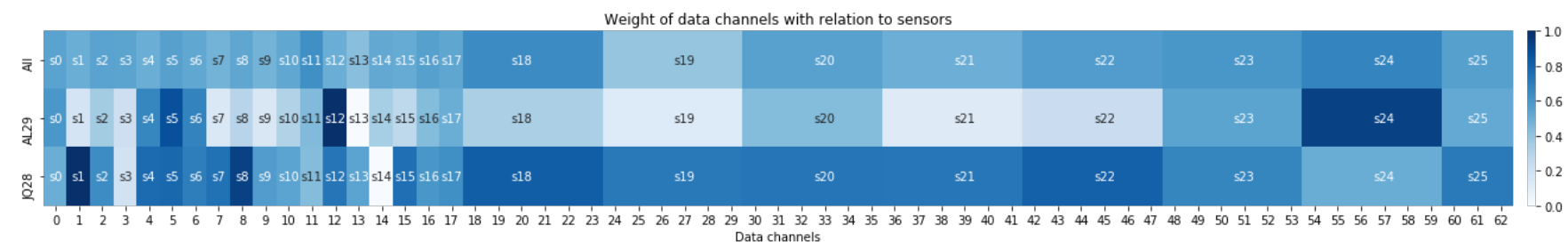

Figure 4: Top presents the $I E$ layer for the general case. Mid, and Bottom presents the $I E$ layer for an individual participant, AL29 and JQ28 respectively. The darker the shade of blue, the higher the weight of the variable for the respective sensor.

In this context, the weights provide a comparison in significance between each sensor, that also takes their cost effectiveness into account.

Equation 1 is presented below to present the idea of the new loss function of the model.

$$
-\sum_{x \in X} p(x) \log (q(x))-\frac{1}{S} \sum_{S}^{S} \operatorname{cost}(s),
$$

where $X$ is the gesture classes and $S$ is the sensors.

For equation $1,-\sum_{x \in X} p(x) \log (q(x))$ is the cross entropy for the multi-class classification. And $\frac{1}{S} \sum_{S}^{S} \cos t(s)$ is the average of the cost function for the sensors. The cost function we utilise for this instance is derived through interpolation of what it would cost to keep a sensor running per hour. It is by no means absolute, but it provides enough to represents the relative cost of each sensor.

\subsection{Results}

The baselines were produced from 10 epochs of 1 st phase training. Then the baseline were used and trained with 2 nd phase for half the amount of epochs from previously, in this case: 5 epochs, to avoid noise in the importance estimates. We then verify the estimates by training a reduced model, with a $10 \%$ increment in reduction of sensors up to $60 \%$. The performance from such were then compared with the performance of the baseline, in terms of TPR of each gesture.

To reiterate from before, the selection of sensors provided by the method is considered valid if a new model with a reduce input is able to retain the same range of results in the metric scores and TPR in the gesture classes. This process of verification is performed in $10 \%$ decrements from the total number of sensors. With the methods explained in 4.1.2, the results of verification regarding the first method, not considering the cost of sensors, is presented below.

Comparing the generic case with personalized when optimizing for accuracy only, the sensors they remove and the performance they attain differs. This is visible from Figure 5, where the results of a subset of participants are available. Only a subset of participants are showcased here due to the limitation of space. The results show a comparison between the TPR of the gesture classes with each $10 \%$ decrements in the total number of sensors used for the models.

Regarding the second method mentioned, to optimise based on prediction of gestures and minimising cost of sensors used, the results are presented in Figure 6. At first sight, it is evident that taking this greedy approach retains similar TPR at the various removal levels as for the case of only considering accuracy. The personalised version tends to result in better TPR than the generic version for some participants, such as JQ28 or YW13.

As evident from the results from Figure 5 and Figure 6, the TPR begin to decline significantly when more than $30-40 \%$ of sensors are removed from the total number of sensors. But for majority of the models with less than $30-40 \%$ of sensors removed, they have managed to retain the same range of TPR despite the removal, which reinforce that the method works. The reduction in cost for different percentages and an average cost reduction is presented in Table 2 and Table 3.

For the specific selection of sensors used and removed, this can be derived from consulting Figure 7. From the results, it is possible to trace each data channel back to the responsible sensor. For the generic case, the sensors removed flex sensor at the base of the ring finger and the pinky. The pressure sensor of the middle finger, as well as the IMU of the middle finger and the pinky. Comparing the generic case and the two specific personalised case, the sensors removed for $A L 29$ are the pressure sensor and IMU of the middle finger, and the IMU of the pinky. And for $7 Q 28$, the sensors removed are the flex sensors at the base and tip of the thumb, the pressure sensor of the middle and ring finger, and the IMU on the wrist. Between $A L 29$ and $\mathcal{F} Q 28$, the flex sensor at the base of the index finger was always removed. Across the participants, $A L 29, \mathcal{F} Q 28$, and the generic case, the pressure sensor of the middle finger was removed consistently.

A comparison is made between the objective of prediction accuracy, and both accuracy-and-cost. It is found that when $40 \%+$ of sensors are removed, both the specific and generic case generally perform better, in terms of TPR for each class, with the objective of accuracy-and-cost. Here we use the results of $\mathcal{F} 28$ for comparison, and we only consider a minimum increase of $5 \%$ as improvement for a gesture class. When the generic case is applied at $40 \%$ removal, accuracy and cost is able to improve the 9 gesture classes with a median of $14.9 \%$, compared to the increase of 3 classes with a median of $10.6 \%$ in accuracy only. In the personalised case, the same can be said for 7 classes with a median of $30 \%$, compared to 4 classes with a median of $10.8 \%$.

Regarding whether a personalised setup is better than the general setup, this is found to be dependent on the participants. For a removal at $50 \%$, the specific case of $Y W 13$ is found to be better than the general case for 15 classes with a median of $21.2 \%$, compared to an improvement of 5 classes with a median of $9.5 \%$. Where as for PC29, the generic case is better for 15 classes with a median of $12.9 \%$, compared to 8 classes with a median of $37.3 \%$ for the specific case. Again the same minimum increase is necessary to be considered an improvement of a class. 

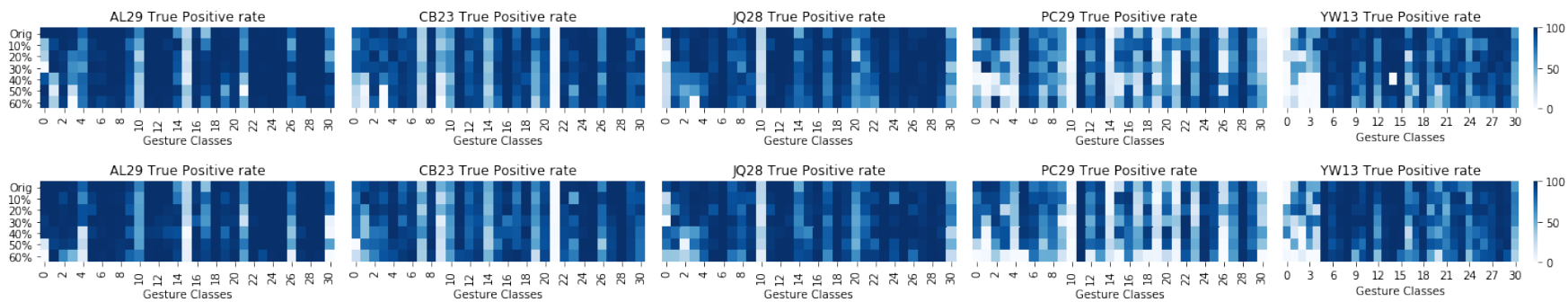

Figure 5: Results regarding the generic case, Top, or the specific case, Bottom, for a subset of participants where the loss function of the model focuses on prediction accuracy. The subset of participants is: AL29, CB23, JQ28, PC29, and YW13

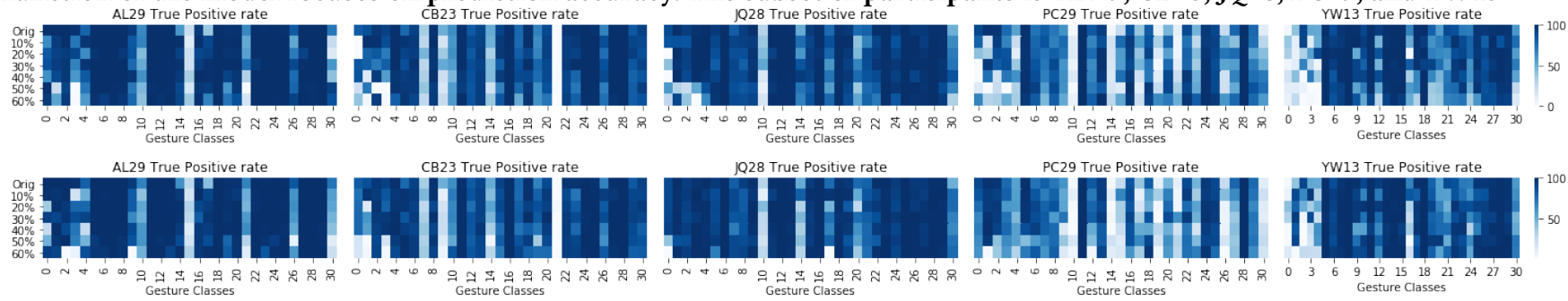

Figure 6: Results regarding the generic case, Top, or the specific case, Bottom, for the same subset of participants used in Figure 5 where the loss function of the model focuses on prediction accuracy and cost of sensors.
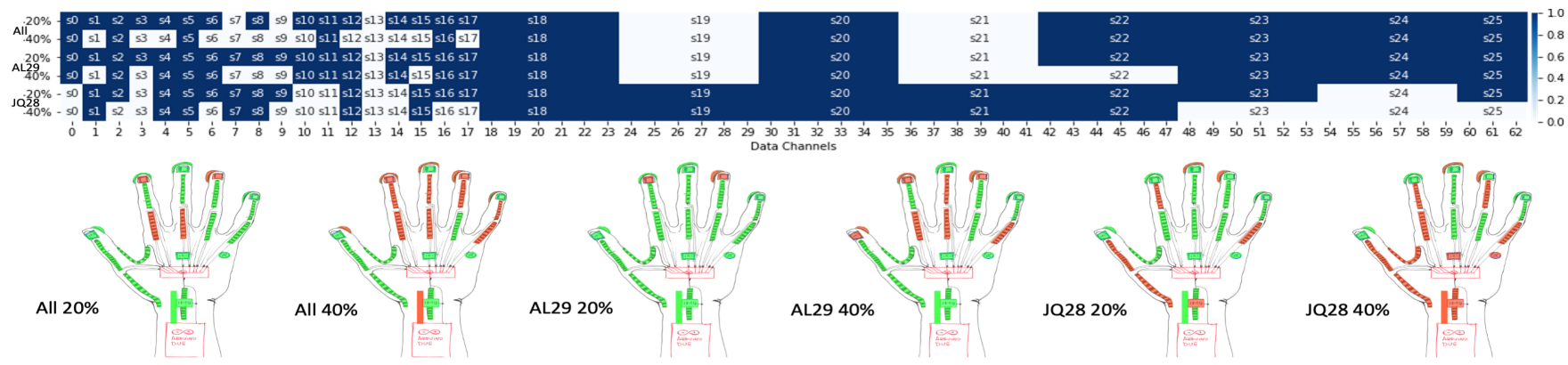

Figure 7: Generic removal at $20 \%$ and $40 \%$ (Top 2). Specific removal at $20 \%$ and $40 \%$ respectively for selected participants: AL29 (Mid 2) and JQ28 (Bottom 2). Below that is a graphical presentation of the setups.

\begin{tabular}{|c|c|c|c|c|c|c|}
\hline \multicolumn{7}{|c|}{ Percentage of usage and reduction in cost for the generic case } \\
\hline \multirow{2}{*}{ Participants } & \multicolumn{2}{|c|}{$10 \%$ reduction } & \multicolumn{2}{|c|}{$20 \%$ reduction } & \multicolumn{2}{|c|}{$30 \%$ reduction } \\
\hline & $\begin{array}{l}\text { Cost } \\
\text { reduction \% }\end{array}$ & $\begin{array}{l}\text { Actual \% of sensors } \\
\text { used }\end{array}$ & $\begin{array}{l}\text { Cost } \\
\text { reduction \% }\end{array}$ & $\begin{array}{l}\text { Actual \% of sensors } \\
\text { used }\end{array}$ & $\begin{array}{l}\text { Cost } \\
\text { reduction \% }\end{array}$ & $\begin{array}{l}\text { Actual \% of sensors } \\
\text { used }\end{array}$ \\
\hline \multirow[t]{2}{*}{ Generic Case } & 6.02 & 90.5 & 19.5 & 76.2 & 26.9 & 71.4 \\
\hline & \multicolumn{2}{|c|}{$40 \%$ reduction } & \multicolumn{2}{|c|}{$50 \%$ reduction } & \multicolumn{2}{|c|}{$60 \%$ reduction } \\
\hline Generic Case & 47.9 & 60.3 & 59.0 & 47.6 & 73.4 & 39.7 \\
\hline
\end{tabular}

Table 2: Percentage of removal in cost and sensors used for the Generic case.

\section{SUBGROUP PERSONALISATION}

It has been demonstrated how the method can be applied to all the gesture classes to create a general or a specifically personalised design for the glove. This can be further developed to specialise the glove for a subset of gestures, and subsequently select a portion of sensors for that subset.

The subset of gestures chosen is, 6:Come Here, 9:Down, 10:Go Away, 21:Swipe Left, 22:Swipe Right, 23:Swipe Up, 25:Thumbs Down and 26:Thumbs Up, which may serve as a natural multimedia flow control. The results of the importance estimates and the $10 \%$ decrements for both the generic and specific case are shown in Figure 8.

The results support that not only is the method capable of personalising the glove for a generic case or a specific individual. But furthermore, it can also be specialised for a specific subset of gesture as shown with no significant change to the architecture and 


\begin{tabular}{|c|c|c|c|c|c|c|}
\hline \multicolumn{7}{|c|}{ Percentage of usage and reduction in cost } \\
\hline \multirow{2}{*}{ Participants } & \multicolumn{2}{|c|}{$10 \%$ reduction } & \multicolumn{2}{|c|}{$20 \%$ reduction } & \multicolumn{2}{|c|}{$30 \%$ reduction } \\
\hline & $\begin{array}{l}\text { Cost } \\
\text { reduction \% }\end{array}$ & $\begin{array}{l}\text { Actual \% of sensors } \\
\text { used }\end{array}$ & $\begin{array}{l}\text { Cost } \\
\text { reduction } \%\end{array}$ & $\begin{array}{l}\text { Actual \% of sensors } \\
\text { used }\end{array}$ & $\begin{array}{l}\text { Cost } \\
\text { reduction \% }\end{array}$ & $\begin{array}{l}\text { Actual \% of sensors } \\
\text { used }\end{array}$ \\
\hline AL29 & 8.46 & 88.9 & 16.9 & 77.8 & 22.9 & 68.3 \\
\hline PC29 & 11.0 & 87.3 & 21.0 & 81.0 & 29.4 & 69.8 \\
\hline \multirow[t]{2}{*}{ JQ28 } & 14.9 & 90.5 & 25.9 & 77.8 & 40.5 & 73.0 \\
\hline & \multicolumn{2}{|c|}{$40 \%$ reduction } & \multicolumn{2}{|c|}{$50 \%$ reduction } & \multicolumn{2}{|c|}{$60 \%$ reduction } \\
\hline AL29 & 30.4 & 63.5 & 38.9 & 52.4 & 38.9 & 52.4 \\
\hline PC29 & 52.4 & 58.7 & 60.9 & 47.6 & 69.4 & 36.5 \\
\hline JQ28 & 61.9 & 49.0 & 62.4 & 52.4 & 64.5 & 39.7 \\
\hline
\end{tabular}

Table 3: Percentage of removal in cost and sensors removed for the Specific case.
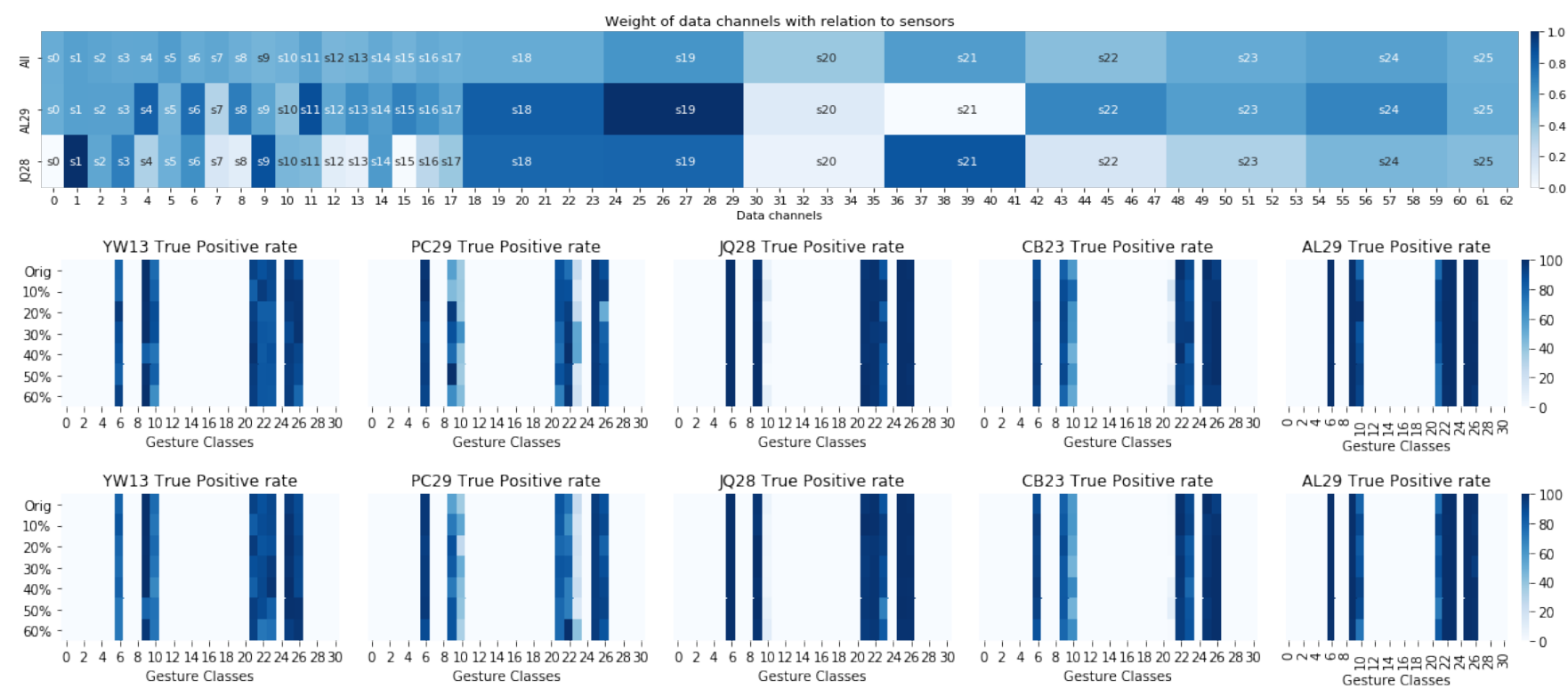

Figure 8: Top shows the importance estimate for the general case, $A L 29$, and JQ28. Followed by the $10 \%$ decrements of the same subset of participants, mentioned in Figure 5, in the case of generic, Middle, and specific, Bottom.

training. Looking at the figures of the weights in the importance estimate, Figure 4 and 8, the weights of the importance estimate are notably different. But it is also clear that despite having different sets of sensors removed, the new models have shown to be capable of predicting the subset as intended.

\section{DISCUSSION AND OUTLOOK}

We intended to use importance estimates as a means to empirically address personalisation and optimisation of a wearable sensor glove. The idea is that with an importance estimate for features, it is possible to make informed decisions rather than random or heuristic driven choices. The intended goal was to reduce redundant sensors in a glove design whilst retaining prediction accuracy for the gestures. The intention was to inform which gestures can be supported with what range of accuracy when the budget is of a certain amount. It was also intended to investigate how personalising the design differs from a generic design.
$R Q 1$ - (Cost-)effective removal of sensors. Our method ranks channels according to their utility, which is measured in what they can deliver in relation to the prediction accuracy, as well as their monetary cost. The method delivers a ranking that can be used for the selection of a subset of sensors, where new models are trained only with the selected subset and their true positive rates of predictions are compared with the baseline for verification. Figure 5 and 6 , show that it is possible to remove sensors to create a minimal design, as well as a cost effective design.

RQ2- Personalisation. Figure 4 illustrates the difference in importance estimates comparing the generic design with two other users. It can be seen that for different users, the importance of the sensors differs. Depending on the user and level of removal, the personalised cases offer better TPRs for higher removal rate. But some participants exhibit better performance in the generic case. This is something we would like to investigate further.

RQ3-Accuracy vs Cost-Accuracy. Figure 7 illustrates the comparison of setups optimised for accuracy with those optimised for 
costs-and-accuracy. The proposed algorithm manages to issue importance estimates according to the corresponding optimisation, and rank the channels therewith. The target optimisation can be varied independent whether personalising or aiming for a generic solution. Each setup produces varying results with respect to TPR.

In general, this paper offers a novel perspective to use feature importance to analyse, personalise or optimise the design of a wearable glove. To the best of our knowledge, this is the first work presenting such in-depth analysis informed by an interpretable deep learning algorithm. This constitutes a much needed extension to the common methods, for it allows a researcher to actually justify the original design decisions with empirical evidence.

\subsection{Comparison with prior work}

Our feature selection method builds upon that from Yifeng et al.[16] and Ning Gui et al.[7]. But it is significantly different from both. Both previous works only dealt with univariate data or data of images. Our method was designed to work with multivariate time series and sliding windows and offers significant improvements in the feature analysis and the feature selection, allowing it to address a broader set of problems as evidenced by our results.

In Yifeng et al.[16], they manually examined the features to look for any relation between them after training a model. In their case, they have an idea of what to look for due to prior knowledge on the subject and how they prepared their sample. But this prior knowledge on the relation between features is not always readily available. We offer a way to not only identify any relation without prior knowledge, but also rank them in importance.

Ning Gui et al.[7] used an attention network between the input and the model, utilising the correlation between the features to determine which features are important based on their probability. Our method does not require the deployment of an attention network, but a layer of weights, which is easier to implement and less complex to understand.

Our method can accommodate external attributes during feature selection. In our case, it is the cost of sensors. Sensors vary in cost and functionality, but since it is not something reflected inherently within the input, any relation identified would not have been able to include this naturally. We address this issue by introducing a cost function for the sensors, the use of another sparse layer, CIE, for the cost of each sensor, and sharing variables between the two sparse layers, IE and CIE. This makes it possible for feature selection to take cost into account during training, so the model is optimised for the intended task as well as being cost effective.

We also proposed to use the importance estimates with a ranking to formulate the design for a personalised case: an individual participant, or a general case: all participants. Which is something that has not been proposed before.

\subsection{Strengths of the method}

The strength of the method stems from its simplicity. A sparse layer that is initialised to $1 \mathrm{~s}$ between the input, and the first hidden layer can be easily implemented into existing architectures. The same can also be said for extending the method with additional sparse layers, to consider cost or any other relations. The only slight complication is the variable sharing between the layers, which would scale in complexity as more layers are added. The simplicity of the method makes it intuitive to understand without the need of extensive tests of data channels or sensor removal.

\subsection{Limitations of the method}

One limitation of our approach is that it requires the implementation of a design to start off with. And the data collection needs to cover all desired possibilities. Yet, in many cases, part of the original hardware and data collection can be performed through simulation. For cases where the hardware setup is to be replicated many times, the proposed method offers an opportunity to reduce the costs of replicas significantly. In the future, we will investigate the possibility to artificially generate data for gestures out of known sensor readings from empirical evidence.

Even though the IE can be interpreted to remove redundancy based on a ranking at the end of training. The full understanding of IE during training is still incomplete. For example, if we expect IE to function as a factor for the input during training, where when IE is $1,1 *$ input, the full input is utilised. And when IE is $0,0 *$ input, the input is ignored. This line of reasoning becomes problematic when IE becomes negative, $-x *$ input, or when it is greater than 1 , $x *$ input. In the proposed method, we avoid this by normalising the weights to $0-1$. But this makes it necessary to evaluate the weights relative to one another. Thus, an isolated weight could not be evaluated without reference, i.e., cannot use the raw value of one channel to determine its significance.

\subsection{Applications in the Design of Wearables}

Smart garments and wearables are designed to deliver information on complex events beyond raw data. For example, various approaches of human activity recognition use IMUs mounted in different body parts. With our method, it is possible to analyse the contribution of each IMU for the classification of activities. This could help with deciding which IMU to include in the production version. Reducing IMUs also reduces wiring, which is a challenge in wearables.

Furthermore, our approach offers a utility optimisation mechanism. It can analyse the utility of sensors with regards to model performance as well as additional constraint, which we have demonstrated with the cost of sensors. With this, the loss function can be extended which allows for feature selection to take the additional constraint into account.

Examples of constraints are: Stability, how reliable are sensor readings or how often do they malfunction. Energy consumption, Weight of sensors, etc. The constraints that can be used are only limited by whether a function with regards to the sensors can be formulated.

In practice, our method also offers a mechanism for empirical validation. Wearables and smart sensors are engineered with various sensors according to some heuristic or prior knowledge (e.g., biomechanics). But the design is hardly questioned once the accuracy for the task is achieved. Our method offers the ability to interpret the significance of a sensor in comparison with the others. 


\section{CONCLUSIONS}

To conclude, a method for reducing hardware complexity whilst maintaining performance with consideration of prediction accuracy and cost has been proposed. This has been shown to be achievable through using interpretability mechanisms in deep learning, specifically the use of importance estimate for the data channels within the data. The estimates have also been expanded upon in terms of feature analysis and feature selection. Regarding feature analysis, the novel approach that was suggested offers a way of ranking each data attribute by their significance in relation to the classification accuracy, or in relation to both the accuracy and a defined cost function. And regarding feature selection, it has been expanded upon with the sharing of variables across additional importance estimate layers, which allows for the inclusion of some form of cost representation regarding the sensors. The cost representation can refer to energy, economical costs or any other aspect that can be numerically attributed to sensors.

Through the results, it is evident that the method can remove sensors, which removes data channels from the input, and is still able to produce models with a decent range of true positive rate for gesture classes. This directly reduces the cost of the hardware, which achieves the goal of reducing hardware complexity whilst maintaining performance that is desired. The method is not without limits, as it needs a good basis of existing configuration in order to perform well. However as discussed previously, the strength of the method is in its simplicity. It can configure and adapt to many data set or architecture. It is also fairly extensible from its functionality where it can take different design decisions in engineering into account. To the best of our knowledge, this is the first work using an explainable deep learning to analyse a wearable design. We hope other researchers will find it useful and adapt our methodology in their design.

\section{ACKNOWLEDGMENTS}

This work was partially funded by AVL List GmbH. Know-Center is funded within the Austrian COMET Program - Competence Centers for Excellent Technologies - managed by the Austrian Research Promotion Agency FFG.

\section{REFERENCES}

[1] R. G. Baraniuk. 2007. Compressive Sensing [Lecture Notes]. IEEE Signal Processing Magazine 24, 4 (July 2007), 118-121. https://doi.org/10.1109/MSP.2007.4286571

[2] Juli Camps, Albert Sam, Mario Martn, Daniel Rodrguez-Martn, Carlos PrezLpez, Joan M. Moreno Arostegui, Joan Cabestany, Andreu Catal, Sheila Alcaine, Berta Mestre, Anna Prats, Maria C. Crespo-Maraver, Timothy J. Counihan, Patrick Browne, Leo R. Quinlan, Gearid Laighin, Dean Sweeney, Hadas Lewy, Gabriel Vainstein, Alberto Costa, Roberta Annicchiarico, ngels Bays, and Alejandro Rodrguez-Molinero. 2018. Deep Learning for Freezing of Gait Detection in Parkinsons Disease Patients in Their Homes Using a Waist-worn Inertial Measurement Unit. Know.-Based Syst. 139, C (Jan. 2018), 119-131. https://doi.org/10.1016/j.knosys.2017.10.017

[3] Salvador Cobos, Manuel Ferre, M. Ángel Sánchez-Urán, Javier Ortego, and Rafael Aracil. 2010. Human hand descriptions and gesture recognition for object manipulation. Computer Methods in Biomechanics and Biomedical Engineering 13, 3 (2010), 305-317. https://doi.org/10.1080/10255840903208171 arXiv:https://doi.org/10.1080/10255840903208171 PMID: 20146129.

[4] G. Devineau, F. Moutarde, W. Xi, and J. Yang. 2018. Deep Learning for Hand Gesture Recognition on Skeletal Data. , 106-113 pages. https://doi.org/10.1109/ FG.2018.00025

[5] L. Dipietro, A. M. Sabatini, and P. Dario. 2008. A Survey of Glove-Based Systems and Their Applications. Trans. Sys. Man Cyber Part C 38, 4 (July 2008), 461-482. https://doi.org/10.1109/TSMCC.2008.923862
[6] Fabio Fumarola, Anna Ciampi, Annalisa Appice, and Donato Malerba. 2009. A Sliding Window Algorithm for Relational Frequent Patterns Mining from Data Streams, Vol. 5808. 385-392. https://doi.org/10.1007/978-3-642-04747-3_30

[7] Ning Gui, Danni Ge, and Ziyin Hu. 2019. AFS: An Attention-based mechanism for Supervised Feature Selection. CoRR abs/1902.11074 (2019). arXiv:1902.11074 http://arxiv.org/abs/1902.11074

[8] Isabelle Guyon and André Elisseeff. 2003. An introduction to variable and feature selection. Fournal of machine learning research 3, Mar (2003), 1157-1182.

[9] Sepp Hochreiter and Jürgen Schmidhuber. 1997. Long Short-Term Memory. Neural Comput. 9, 8 (Nov. 1997), 1735-1780. https://doi.org/10.1162/neco.1997.9. 8.1735

[10] Sara Hooker, D. Erhan, P. Kindermans, and Been Kim. 2019. A Benchmark for Interpretability Methods in Deep Neural Networks. In NeurIPS.

[11] Chung-Lin Huang and Wen-Yi Huang. 1998. Sign language recognition using model-based tracking and a 3D Hopfield neural network. Machine Vision and Applications 10, 5 (01 Apr 1998), 292-307. https://doi.org/10.1007/s001380050080

[12] Holger Kenn, Friedrich Van Megen, and Robert Sugar. 2007. A glove-based gesture interface for wearable computing applications. In Applied Wearable Computing (IFAWC), 2007 4th International Forum on. 1-10.

[13] Arun Kulshreshth and Joseph J. LaViola, Jr. 2015. Exploring 3D User Interface Technologies for Improving the Gaming Experience. In Proceedings of the 33rd Annual ACM Conference on Human Factors in Computing Systems (Seoul, Republic of Korea) (CHI '15). ACM, New York, NY, USA, 125-134. https://doi.org/10.1145/ 2702123.2702138

[14] P Kumar, J Verma, and Shitala Prasad. 2012. Hand data glove: A wearable realtime device for human-computer interaction. International fournal of Advanced Science and Technology 43 (01 2012), 15-26.

[15] Joseph J. LaViola, Jr. 1999. A Survey of Hand Posture and Gesture Recognition Techniques and Technology. Technical Report. Providence, RI, USA.

[16] Yifeng Li, Chih-Yu Chen, and Wyeth W. Wasserman. 2015. Deep Feature Selection: Theory and Application to Identify Enhancers and Promoters. In Research in Computational Molecular Biology, Teresa M. Przytycka (Ed.). Springer International Publishing, Cham, 205-217.

[17] Yang LI, Jin HUANG, Feng TIAN, Hong-An WANG, and Guo-Zhong DAI. 2019. Gesture interaction in virtual reality. Virtual Reality \& Intelligent Hardware 1, 1 (2019), 84 - 112. https://doi.org/10.3724/SP.J.2096-5796.2018.0006

[18] G. Luzhnica, J. Simon, E. Lex, and V. Pammer. 2016. A sliding window approach to natural hand gesture recognition using a custom data glove. In 2016 IEEE Symposium on $3 D$ User Interfaces (3DUI). 81-90. https://doi.org/10.1109/3DUI. 2016.7460035

[19] Tiago Martins, Christa Sommerer, Laurent Mignonneau, and Nuno Correia. 2008. Gauntlet: a wearable interface for ubiquitous gaming. In MobileHCI '08: Proceedings of the 10th international conference on Human computer interaction with mobile devices and services. ACM Request Permissions, New York, New York, USA, 367.

[20] Kouichi Murakami and Hitomi Taguchi. 1991. Gesture Recognition Using Recurrent Neural Networks. In Proceedings of the SIGCHI Conference on Human Factors in Computing Systems (New Orleans, Louisiana, USA) (CHI '91). ACM, New York, NY, USA, 237-242. https://doi.org/10.1145/108844.108900

[21] P. Neto, D. Pereira, J. Norberto Pires, and A.P. Moreira. 2013. Real-time and continuous hand gesture spotting: An approach based on artificial neural networks. In Robotics and Automation (ICRA), 2013 IEEE International Conference on. 178-183. https://doi.org/10.1109/ICRA.2013.6630573

[22] Francisco Ordóñez and Daniel Roggen. 2016. Deep Convolutional and LSTM Recurrent Neural Networks for Multimodal Wearable Activity Recognition. Sensors 16, 1 (Jan 2016), 115. https://doi.org/10.3390/s16010115

[23] Cemil Oz and Ming C. Leu. 2011. American Sign Language word recognition with a sensory glove using artificial neural networks. Engineering Applications of Artificial Intelligence 24, 7 (2011), 1204 - 1213. https://doi.org/10.1016/j.engappai. 2011.06.015 Infrastructures and Tools for Multiagent Systems.

[24] Gang Ren and Eamonn O'Neill. 2013. Freehand Gestural Text Entry for Interactive TV. In Proceedings of the 11th European Conference on Interactive TV and Video (Como, Italy) (EuroITV '13). ACM, New York, NY, USA, 121-130. https://doi.org/ $10.1145 / 2465958.2465966$

[25] M. Romaszewski, P. Glomb, and P. Gawron. 2014. Natural hand gestures for human identification in a Human-Computer Interface. In Image Processing Theory, Tools and Applications (IPTA), 2014 4th International Conference on. 1-6. https: //doi.org/10.1109/IPTA.2014.7001997

[26] Debaditya Roy, K. R. Murty, and C. K. Mohan. 2015. Feature selection using Deep Neural Networks. 2015 International foint Conference on Neural Networks (IFCNN) (2015), 1-6.

[27] David J. Sturman and David Zeltzer. 1994. A Survey of Glove-based Input. IEEE Comput. Graph. Appl. 14, 1 (Jan. 1994), 30-39. https://doi.org/10.1109/38.250916

[28] Tomoichi Takahashi and Fumio Kishino. 1991. Hand Gesture Coding Based on Experiments Using a Hand Gesture Interface Device. SIGCHI Bull. 23, 2 (March 1991), 67-74. https://doi.org/10.1145/122488.122499

[29] Tsung-Han Tsai, Chih-Chi Huang, and Kung-Long Zhang. 2015. Embedded virtual mouse system by using hand gesture recognition. In Consumer Electronics 
- Taiwan (ICCE-TW), 2015 IEEE International Conference on. 352-353. https: //doi.org/10.1109/ICCE-TW.2015.7216939

[30] Andrea Utley and Sarah. Astill. 2018. Motor Control Learning and Development: instant notes, 2nd. Taylor and Francis.

[31] A. Verikas and M. Bacauskiene. 2002. Feature selection with neural networks. Pattern Recognition Letters 23, 11 (2002), 1323 - 1335. https://doi.org/10.1016/ S0167-8655(02)00081-8

[32] J. Weissmann and R. Salomon. 1999. Gesture recognition for virtual reality applications using data gloves and neural networks. In Neural Networks, 1999. IfCNN '99. International foint Conference on, Vol. 3. 2043-2046 vol.3.

[33] Deyou Xu. 2006. A Neural Network Approach for Hand Gesture Recognition in Virtual Reality Driving Training System of SPG. In Pattern Recognition, 2006. ICPR 2006. 18th International Conference on, Vol. 3. 519-522.

[34] Xu Zhang, Xiang Chen, Wen-hui Wang, Ji-hai Yang, Vuokko Lantz, and Kongqiao Wang. 2009. Hand Gesture Recognition and Virtual Game Control Based on 3D Accelerometer and EMG Sensors. In Proceedings of the 14th International Conference on Intelligent User Interfaces (Sanibel Island, Florida, USA) (IUI '09).
ACM, New York, NY, USA, 401-406. https://doi.org/10.1145/1502650.1502708

[35] L. Zhao, Q. Hu, and W. Wang. 2015. Heterogeneous Feature Selection With Multi-Modal Deep Neural Networks and Sparse Group LASSO. IEEE Transactions on Multimedia 17, 11 (2015), 1936-1948.

[36] Yang Zheng, Yu Peng, Gang Wang, Xinrong Liu, Xiaotong Dong, and Jue Wang. 2016. Development and evaluation of a sensor glove for hand function assessment and preliminary attempts at assessing hand coordination. Measurement 93 (2016), 1 - 12. https://doi.org/10.1016/j.measurement.2016.06.059

[37] Thomas G. Zimmerman, Jaron Lanier, Chuck Blanchard, Steve Bryson, and Young Harvill. 1987. A Hand Gesture Interface Device. In Proceedings of the SIGCHI/GI Conference on Human Factors in Computing Systems and Graphics Interface (Toronto, Ontario, Canada) (CHI '87). ACM, New York, NY, USA, 189-192. https://doi.org/10.1145/29933.275628

[38] Jacek M. Zurada, Aleksander Malinowski, and Shiro Usui. 1997. Perturbation method for deleting redundant inputs of perceptron networks. Neurocomputing 14, 2 (1997), 177 - 193. https://doi.org/10.1016/S0925-2312(96)00031-8 